\title{
SOME RELATIONSHIPS BETWEEN PERIPHERAL VASOMOTOR AND E.E.G. CHANGES
}

\author{
BY \\ BRIAN ACKNER and G. PAMPIGLIONE \\ From the Institute of Psychiatry, Maudsley Hospital, London, S.E.5
}

The influence of emotional and physical stimuli on the variations in the volume of the extremities was recognized as far back as 1881 by Mosso. Subsequent workers using arm and finger plethysmography noted that the vasoconstrictor response to various stimuli was not only present during wakefulness but could also occur during sleep (Hallion and Comte, 1894; Howell, 1897; Brodmann, 1902; Berger, 1904; Shepard, 1906, 1914; Wiersma, 1913; Uhlenbruck, 1924). The increase in spontaneous fluctuations in the plethysmographic curve during drowsiness and early sleep was noted by Hallion and Comte (1894) and thought by Shepard $(1906,1914)$ to be due to Traube-Hering blood pressure waves. Later workers offered different explanations. Ingram (1936) and Burton (1939) considered the fluctuations to be serving temperature control, Abramson and Katzenstein (1941) regarded them as spontaneous peripheral changes, whilst Neumann, Lhamon, and Cohn (1944) held that they were a reflection of variations in sympathetic tone in relaxing subjects. Ackner (1956b) has shown that, when the experimental conditions are suitably controlled, the onset of sleep is associated with an increase in the finger pulse volume in anxious subjects. This is presumably due to the release of stress vasoconstriction, for such vasodilatation does not occur in the relaxed subject. The physiological and emotional factors related to the finger plethysmographic vasoconstrictor response have recently been reviewed by Ackner (1956a).

It emerges from previous work that the vasoconstrictor response is not dependent on wakefulness, though the response disappears when deep levels of sleep are reached. Until recently only Jung $(1939,1954)$ attempted further investigation of the relationship between peripheral vasomotor changes and levels of "consciousness" by means of combined plethysmographic and electroencephalographic (E.E.G.) studies. Jung recorded simultaneously the E.E.G., electrocardiogram, galvanic skin reflex, finger plethysmograph, and respiration during wakefulness and different stages of sleep. He stated that "the autonomic responses associated with arousal do not change in different states of consciousness, except that conditioning and expectation of the stimulus tend to suppress and diminish the reactions in the waking state ". In a preliminary communication (Ackner and Pampiglione, 1955) we reported findings at variance with this conclusion, and in this paper we shall describe in more detail some relationships between peripheral vasomotor and E.E.G. changes. In a later communication the relationship between changes in other physiological variables will be considered.

\section{E.E.G. Changes from Alertness to Sleep}

The E.E.G. features associated with the transitional stages from full alertness to light and deep sleep in man are well known since the work of Loomis, Harvey, and Hobart (1937, 1938), Davis, Davis, Loomis, Harvey, and Hobart (1938, 1939), Blake and Gerard (1937), Kleitman (1939), and others.

Recently, the E.E.G. changes following sensory stimuli during sleep, in patients and normal subjects, have been further investigated by Brazier (1949, 1954), Li, Jasper, and Henderson (1952), Pampiglione (1952a, 1953), Pampiglione and Martin (1954), and Lairy-Bounes and Fischgold (1953). Some work on this subject has been reported from the U.S.S.R., but the relevant literature is not easily available.

A number of E.E.G. patterns have been considered to be associated with particular "sleep levels". However, the variability of the E.E.G. in normal subjects, from alertness to sleep, is wide with much individual diversity.

In the interpretation of our findings we have used, as broad correlates of alertness and sleep levels, the following fairly well recognizable E.E.G. changes in adults:-

(1) Full Alertness with Active Interest.-Generalized low amplitude activity.

(2) Alertness with Reduced Interest.-Appearance of fluctuating rhythmic activity at 8 to $13 \mathrm{c} / \mathrm{sec}$. (alpha rhythms) mainly over the posterior half of the head. 
(3) Minimal Alertness or Drowsiness.-Intermittent, short-lived appearance of alpha rhythms on a background of generalized low amplitude 4 to $7 \mathrm{c} / \mathrm{sec}$. activity.

(4) Light Sleep.-Occasional and sometimes periodic runs of rhythmic activity at 12 to $15 \mathrm{c} / \mathrm{sec}$. in the form of "spindles" (best seen in the anterior two-thirds of the head) on a generalized background of slower activity.

(5) Medium Depth of Sleep.-Slow waves usually generalized, but not obviously rhythmic, with rare occurrence of " spindles".

(6) Fairly Deep Sleep.-Generalized slow waves often rhythmic and sometimes synchronous in various areas.

The E.E.G. change that follows a stimulus during sleep is described as a "K-complex". This appears as a well recognizable change in amplitude and frequency often in the form of one, or more, large diphasic slow waves, which may be accompanied or replaced by a run of rhythmic activity (" spindles " or even alpha rhythms). This change may be short lived, but sometimes (and possibly in relation to some properties of the stimulus) the E.E.G. may change for a while to a different " level " of activity. The modifications in the E.E.G. after a stimulus may not always be associated with a recognizable change in the subject's appearance, even when very marked E.E.G. changes occur.

The occurrence of $\mathrm{K}$-complexes has been studied by the various workers previously mentioned. The $\mathrm{K}$-complex, although considered by some to represent a "partial awakening", might be related instead to mechanisms concerned with the maintenance or protection of sleep. The K-complex is a non-specific response in the E.E.G. as it can be induced by a variety of stimuli. However, if stimuli are repeated several times " adaptation phenomena" occur (Pampiglione, 1952b), with diminution or non-appearance of the $\mathrm{K}$-complex after a number of stimuli. Commonly," spontaneous "E.E.G. changes are seen during sleep, morphologically very similar to K-complexes evoked by external stimuli. Although not clearly proved, it is possible that unnoticed or " internal " stimuli, such as extrasystoles, changes in respiration, the movement of a limb, borborygmi, etc., and perhaps dreams, might be related to the appearance of such changes. For descriptive convenience we shall name these "spontaneous $K$ complexes".

During the waking period, both the reappearance and the blocking of the alpha rhythms after a stimulus may be comparable with the occurrence of a Kcomplex following a stimulus during sleep. The "spontaneous" appearance or disappearance of alpha rhythms seems to bear a similar relationship to the occurrence of " spontaneous K-complexes" during sleep.
The Plethysmographic Vasoconstrictor Response

In this study the peripheral vascular changes were recorded by means of a finger plethysmograph. As the blood supply to the finger does not include vessels to muscular tissue, mainly cutaneous vasomotor reactions are recorded using this technique. There is no evidence of dilator fibres in the digits and the innervation is entirely sympathetic in origin (Warren, Walter, Romano, and Stead, 1942; Arnott and Macfie, 1948; Bridges and Yahr, 1955). A sudden rapid reduction in the amplitude of the pulse volume waves can thus be taken as evidence of vasoconstriction and of increased sympathetic activity along the vasomotor pathways. The pulse volume waves are related to the heart beat but their size is largely dependent on the degree of vascular dilatation. In contrast to the limb plethysmograph, blood pressure changes do not appear to be of much significance in evaluating changes observed in the finger plethysmograph (Burton, 1939; Abramson and Ferris, 1940; Neumann, 1943; Matthes, 1952). After an adequate stimulus, e.g., noise, light, pain, a threat, the pulse and finger volume is decreased, with a latent period of three to four seconds, whatever effective stimulus is employed. This response is absent in the sympathectomized limb. The degree of the reduction of volume following a stimulus is partly related to the pre-existing dilatation and no reflex vasoconstriction can be expected if the vessels are not adequately dilated at the time. The duration and magnitude of the vasoconstrictor response vary considerably and are related both to the intensity of the stimulus and to its significance for the individual. Repetition of the same stimulus during the waking state usually results in a diminution and finally a disappearance of the response.

A similar vasoconstrictor response may occur following a spontaneous deep inspiration with a latency of three to four seconds, although absent in the sympathectomized limb. This response may not always follow a deep voluntary inspiration but, when it does, repetition of deep inspiration tends to reduce the response until it is finally extinguished (Bolton, Carmichael, and Sturup, 1936).

\section{Experimental Procedure}

The technique of multiple simultaneous recording used has already been described by Ackner (1956b). A special feature of the plethysmographic recording was the separation of the pulse and finger volumes changes onto two separate pens. Recording on a single pen always results in the slower finger volume changes altering the base-line and distorting the appearance of the pulse volume changes. Utilizing different time constants the slower gross finger volume changes were sampled and registered by a pen 
marking "pips", the intervals of which lengthened or shortened with increase or decrease in the volume of the finger tip respectively. A separate pen recorded the pulse volume changes. Electroencephalograms were taken with a three-channel amplifier, using electrodes placed on the frontal, central, parietal, and occpital areas of the left side of the head. An audiostimulator was used, capable of delivering a note of $0.5 \mathrm{sec}$. duration with fixed or variable intensity, pitch, and repetition rate.

Recordings were carried out in a room maintained at $22^{\circ} \mathrm{C}$. and under quiet conditions. Most of the apparatus was housed in an adjoining room. Continuous records were taken during the waking state, drowsiness, natural sleep, or oral " seconal"-induced sleep. Satisfactory drowsiness and sleep was achieved in 13 normal control subjects and 40 psychiatric patients suffering from mixed neurotic states. From the control group 13 sleep records were obtained of which 11 were naturally occurring and two were induced by oral "seconal". From the psychiatric patients 47 sleep records were obtained, of which 17 were naturally occurring and 30 were induced by oral " seconal". There was thus a total of 60 records.

\section{Findings}

Resting State and Drowsiness. - The pulse volume waves in an awake and fully alert subject showed little change in amplitude, unless external stimuli or

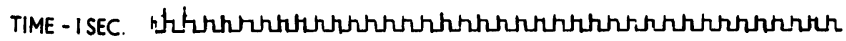

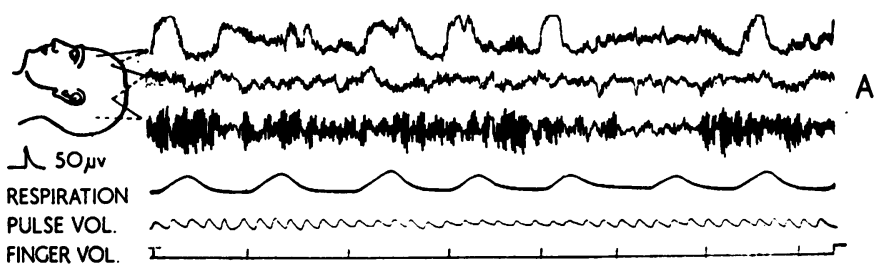

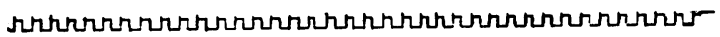

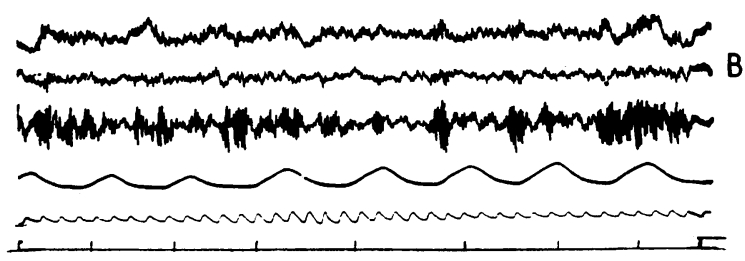

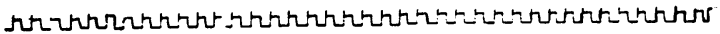

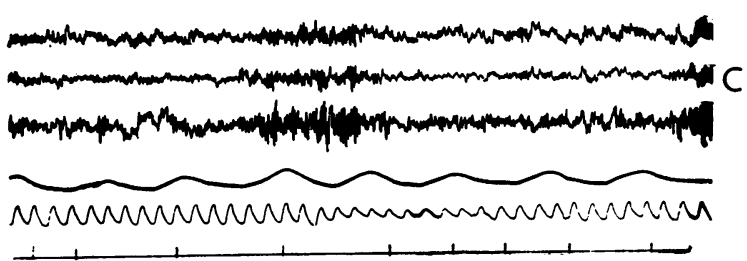

emotional disturbance were present. As the subject began to relax the pulse volume waves showed periods of decrease in amplitude lasting a few seconds. With increasing relaxation and drowsiness these fluctuations often took on a more periodic racter, sometimes occurring two or three times a These were most marked during the early of sleep, and, as sleep deepened, tended to lose periodicity, becoming more episodic in their These phenomena were not so commonly observed in tense, anxious subjects whose pulse volume waves usually remained small and regular in amplitude until early sleep. At this stage, the release of the stress vasoconstrictor factor ulted in a progressive vasodilatation with steady ease in the amplitude of the pulse volume waves. vasoconstrictions tended to become more infrequent as sleep progressed.

With the subject fully alert, though with the eyes hut, the E.E.G. usually showed little or only a derate amount of alpha rhythms which increased as relaxation began. At this stage a stimulus or increased alertness might cause a decrease or disappearance of alpha rhythms for a time. Associated with this change there was often a vasoconstrictor response (about three to four seconds after the blocking of alpha rhythms), and the subsequent return of alpha rhythms was usually followed by a slow vaso-e dilatation, though the latter might be delayed. Spontaneous fluctuations in the amplitude of the pulse volume waves often began when the E.E.G. showed a considerable number of alpha rhythms, but at this stage it was not always possible to observe a correlation between changes in the E.E.G. and changes in the amplitude of the pulse volume waves. With further relaxation and the onset of drowsiness the alpha rhythms intermittently disappeared, and it was at this stage that

FIG. 1-Variations in amplitude of the pulse volume waves while the subject (Mrs. W.) is: (A) Awake-(no clear relationship with the slight variations in amount of alpha rhythms). (B) A wake - (no clear relationship with slightly more marked, but not yet periodic changes in amount of alpha rhythms). (C) Drowsy after " seconal ", gr. 1.5- (vasoconstriction follows the periodic reappearance of alpha rhythms). No constant variations in rate or amplitude of respiration. 
TIME - I SEC

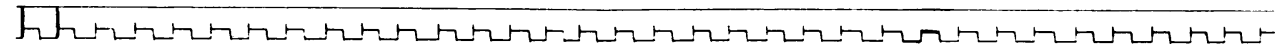

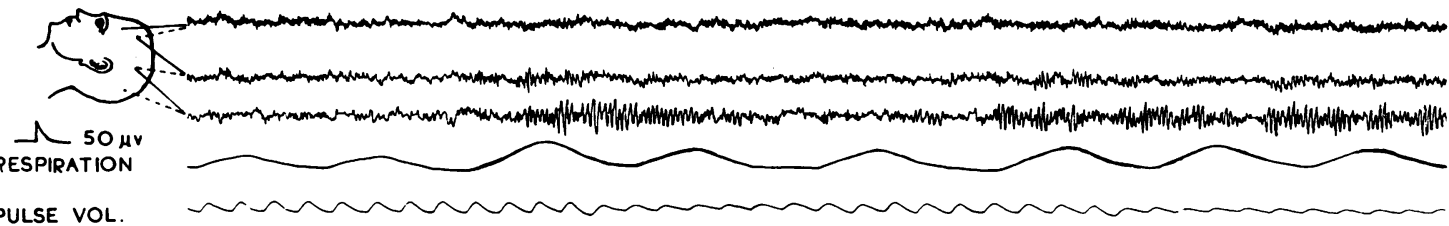

PULSE VOL.

FINGER VOL.

FIG. 2.-Subject (Miss O. M.) drowsy (no drugs): The return of alpha rhythms is followed by diminution of the amplitude of the pulse volume waves on two occasions. On the first occasion there is a change in depth of respiration while on the second occasion there is no change.

the fluctuations in the amplitude of the pulse volume waves tended to become more periodic and to have an increasing association with the E.E.G. changes (Fig. 1). The return of alpha rhythms at this stage was often followed (about three or four seconds later) by a rapid vasoconstriction lasting a few seconds. A gradual vasodilatation followed which was maintained during the subsequent phase of disappearance of alpha rhythms. A subsequent return of alpha rhythms might again be followed by a period of vasoconstriction. For a time periodic reductions in the amplitude of the pulse volume waves were often associated with the reappearance of the alpha rhythms, and as the latter became more infrequent, so did the associated vasoconstrictions (Fig. 2).

Sleep.-During light sleep, "spindles" and some slow activity appear in the E.E.G. while the alpha rhythms are no longer seen. If the alpha rhythms spontaneously reappeared vasoconstriction usually followed. The occasional or periodic appearance of spindles, however, was not associated with changes in the pulse volume waves (Fig. 3). An adequate external stimulus at this stage would cause the reappearance of alpha rhythms followed by a vasoconstrictor response with the usual latency. The morphological characteristics and time relationships of both these E.E.G. and plethysmographic changes were similar whether these were evoked or occurred spontaneously (Fig. 4).

Fig. 3.-Subject (Mr. B.) asleep after " seconal", gr. 3: (A) No change in pulse waves accompanying the appearance of spindles. (B) "Spontaneous" variations in amount of slow waves accompanied by vasoconstriction. (C) "Spontaneous" variations in amount of slow waves not accompanied by vasoconstriction.

FINCER VOL.

As sleep deepened, " spontaneous" K-complexes occurred from time to time in the E.E.G. and these were often associated with a vasoconstrictor response. At this stage of sleep an adequate external stimulus evoked a K-complex in the E.E.G. followed by a vasoconstriction. Again the E.E.G., the plethysmographic changes, and their time relationships were similar whether such changes were evoked or occurred spontaneously (Fig. 5). Sometimes the evoked changes in the E.E.G. were followed some seconds later by " secondary" K-complexes and these were
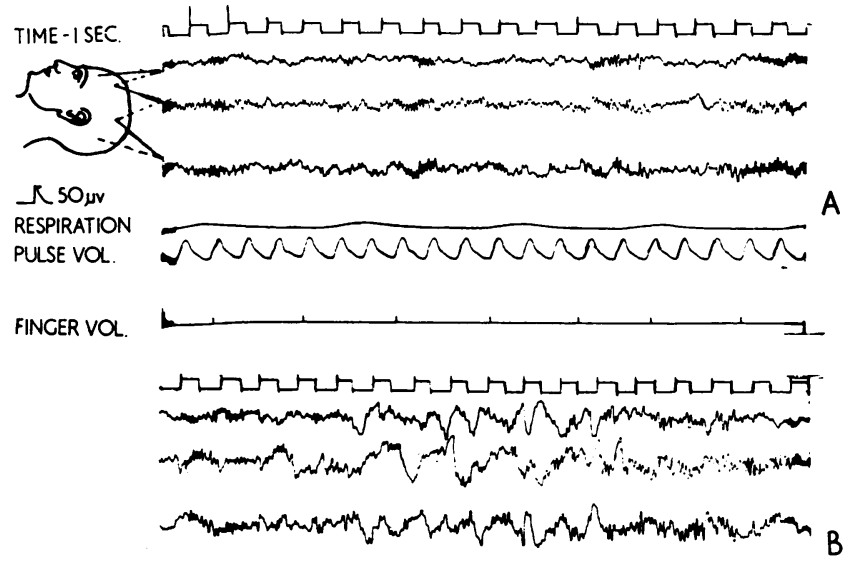

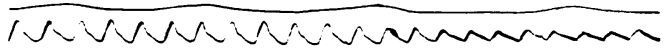

1

$$
\text { }
$$


TIME-I SEC

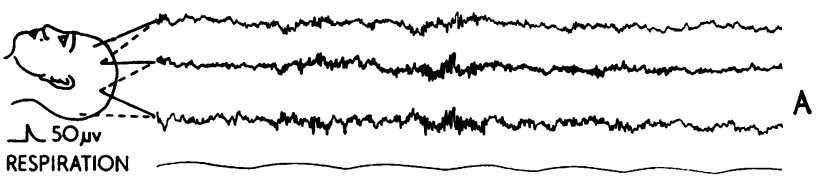

PULSE $\mathrm{VO}$. FINCER VOL.
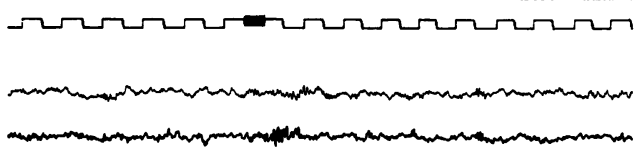

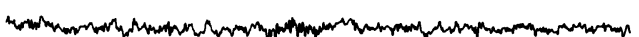

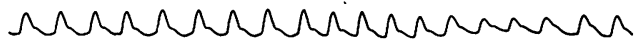

FIG. 4.-Subject (Mrs. C.) drowsy after " seconal” " gr. 3 : (A) “'Spontaneous" return of alpha rhythms followed by vasoconstriction. (B) Evoked return of alpha rhythms is followed by vasoconstriction with a delay similar to the "spontaneous" response in (A) above. (Stimulus indicated by black area on time marker.)

Fig. 5.-Subject (Mrs. S.) asleep after " seconal", gr. 3: (A) Two "spontaneous" K-complexes are followed by vasoconstriction. (B) K-complex elicited by stimulus is followed by vasoconstriction with a delay similar to the " spontaneous" response in (A) above. (Stimulus indicated by black area on time marker.)

TIME-ISEC.
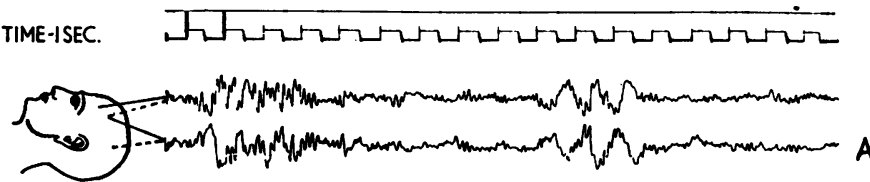

入 $50 \mu v$ RESPIRATION PULSE VOL.

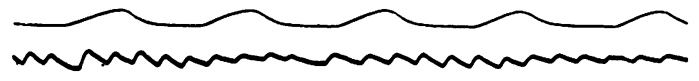

FINGER VOL
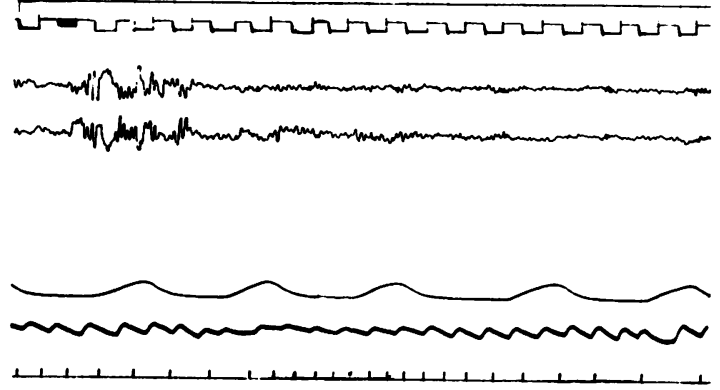

on occasions associated with a further vasoconstriction. Sometimes a "spontaneous" K-complex was followed a few seconds later by one or more further $\mathrm{K}$-complexes and a vasoconstrictor response might be associated with the initial or the subsequent E.E.G. changes or with both. When prolonged E.E.G. changes occurred these might be accompanied by vasoconstriction, but the onset of the latter had a variable time relationship to the onset of the E.E.G. changes.

Repetition of an adequate external stimulus, such as a high-pitched note, at a suitable constant rate, e.g., every 20 seconds, resulted in more complex changes which will be reported in detail in a later communication.

Deep Sleep, Awakening, and Subsequent Drowsiness.-As sleep progressively deepened an external stimulus evoked a less recognizable E.E.G. change and the latter was less often associated with a vaso motor change. Unless the level of sleep was changed by the stimulus a vasoconstrictor response did not usually occur during deep sleep. Awakening with return of alpha rhythms was always followed by a marked vasoconstriction, with subsequenf gradual vasodilatation. When the drowsy post-sleep stage was prolonged, wax ing and waning of alpha rhythms associated with changes in the amplitude of the pulse volume was commonly seen.

\section{Discussion}

External stimuli and other influences causing a change in attitude or alertness of the individual have long been known to cause responses in the E.E.G. and plethysmographic record. An external stimulus will be followed a fraction of a second later by recognizable changes in the E.E.G., such as variations in the alpha rhythms or the appearance of K-complexes, according to the level of alertness or sleep. In the plethysmograph a fairly rapid vasoconstriction occurs with a latency of some three to four seconds following an adequate external stimulus. The E.E.G. and plethysmograph simultaneously recorded show not unexpectedly that these responses, when evoked 
by external stimuli, are related to each other in time. This type of simultaneous recording reveals in addition that certain apparently spontaneous vasoconstrictor changes in the finger are commonly associated with apparently spontaneous E.E.G. changes and follow the latter after some three to four seconds. This is the same latency as that of the evoked responses. Furthermore, the morphology of the " spontaneous" E.E.G. changes preceding the "spontaneous" vasoconstrictions varies according to the level of alertness or sleep in just the same way as do the E.E.G. responses evoked by alerting stimuli. It thus appears probable that many of the apparently spontaneous vasoconstrictions in the plethysmographic record, which have formerly been ascribed to a variety of causes, are really related to fluctuations in the levels of alertness or sleep. Presumably such vasoconstriction of the skin vessels is, as with the evoked changes, part of a bodily alerting reaction, perhaps diverting blood to areas which are becoming mobilized for activity.

A subject's pattern of falling asleep or sleeping may vary according to many factors, such as the state of his digestion, his preoccupations of the moment, the external stimuli applied during testing, and how long he is left to sleep. Thus the same individual's pattern may vary from one occasion to another. Because of this variability, the findings have been presented in a descriptive form rather than assessed quantitatively in terms of their relative appearance. Such results would only be valid for the particular group of subjects observed. Another group composed of similar subjects would no doubt reveal phenomena similar in nature but not necessarily comparable in extent.

The relationships described are not easily recognizable in all records, being difficult to observe in those subjects who are tense before sleep or who fall asleep relatively suddenly and deeply. They are best seen in those subjects who, by relaxing easily, remaining drowsy, or falling asleep slowly, show fluctuations in levels of E.E.G. activity before finally reaching deep sleep. The phenomena occur, however, in both normal subjects and in psychiatric patients, whether drowsiness and sleep occur naturally or are induced by oral " seconal ".

From time to time, and especially during the early stage of sleep, the plethysmographic changes noted above are associated with a change in the character of respiration. That the latter is not the significant factor determining the plethysmographic changes or their relationship to the E.E.G. changes is quite certain, for the E.E.G. and plethysmographic changes described are commonly seen in the absence of any appreciable variation in the respiratory rate, rhythm, or amplitude.

\section{Conclusion}

An external stimulus which will cause, with a latency of three to four seconds, a peripheral vasoconstrictor response tends also to evoke almost immediately in the E.E.G. a disappearance of alpha rhythms, a reappearance of alpha rhythms, or a $\mathrm{K}$-complex, according to whether the subject is awake, drowsy, or in medium sleep respectively.

Peripheral vasoconstrictor changes occurring apparently spontaneously are often preceded (by three to four seconds) by apparently spontaneous disappearance of alpha rhythms, reappearance of alpha rhythms, or a K-complex, according to whether the subject is awake, drowsy, or in medium sleep respectively.

Many apparently spontaneous vasoconstrictor changes are thus preceded by E.E.G. changes with a time relationship and morphology similar to those evoked by alerting stimuli at different stages of wakefulness or sleep.

It thus appears probable that these apparently spontaneous vasoconstrictions are really occurring in relation to spontaneous fluctuations in the levels of alertness or sleep.

\section{Summary}

Simultaneous E.E.G. and plethysmographic recordings were carried out on normal and neurotic subjects during wakefulness and during natural and " seconal "-induced sleep. Changes in alertness or level of "consciousness" were often found to be associated with E.E.G. and plethysmographic changes in a recognizable relationship. The morphology and time relationships of these phenomena were similar, whether the changes in alertness or in level of sleep were evoked or occurred spontaneously. These phenomena were observable both in normal and neurotic subjects, whether drowsiness and sleep occurred naturally or were induced by oral "seconal". Their occurrence appeared to bear a relationship to the state of relaxation of the individual and to the pattern of falling asleep. It is suggested that many of the "spontaneous" peripheral vasoconstrictor changes occurring during relaxation, drowsiness, and early sleep have the same significance as the responses evoked by an alerting stimulus.

Part of this work was supported by a grant from the Research Committee of the Board of Governors of Bethlem Royal and the Maudsley Hospitals to whom the authors are indebted. We should also like to thank Dr. Denis Hill for his encouragement, Mr. Peter St. John Loe for help in development of apparatus, and Mr. W. A. Gurnhill for technical assistance. 


\section{REFERENCES}

Abramson, D. I., and Ferris, E. B. (1940). Amer. Heart J., 19, 541. , and Katzenstein, K. H. (1941). Ibid., 21, 191.

Ackner, B. (1956a). J. Psychosom. Res., 1, 3.

(1956b). Ibid., 1, 21.

and Pampiglione, G. (1955). Electroenceph. clin. Neurophysiol. 7,153

Arnott, W. M., and Macfie, J. M. (1948). J. Physiol. (Lond.), 107, 233.

Berger, H. (1904), Uber die körperlichen Ausserungen psychischer Zustände. Fischer, Jena.

Blake, H., and Gerard, R. W. (1937). Amer. J. Physiol., 119, 692. Bolton, B., Carmichael, E. A., and Sturup, G. K. (1936).' J. Physiol. (Lond.), 86, 83

Brazier, M. A. B. (1949). Electroenceph. clin. Neurophysiol., 1, 195. (1954). In Brain Mechanisms and Consciousness: A Symposium organized by the Council for International Organizations of Medical Sciences, p. 163. Blackwell, Oxford.

Bridges, T. J., and Yahr, M. D. (1955). A.M.A. Arch. Neurol. Psychiat., 74, 534 .

Brodmann, K. (1902). J. Psychol. Neurol. (Lpz.), 1, 10.

Burton, A. C. (1939). Amer. J. Physiol., 127, 437.

Davis, H., Davis, P. A., Loomis, A. L., Harvey, E. N., and Hobart, G. (1938). J. Neurophysiol., 1, 24

- _ (1939). Ibid, 2,500

Hallion, L., and Comte, C. (1894). Arch. Physiol. (Paris), 5 ser. 6, 381 .

Howell, W. H. (1897). J. exp. Med., 2, 313.

Ingram, P. W. (1936). Edinb. med. J., 43, 672.
Jung, R. (1939). Nervenarzt, 12, 169. (1954). In Brain Mechanisms and Consciousness, p. 310. Oxford.

Kleitman, N. (1939). Sleep and Wakefulness. University of Chicago Press, Chicago.

Lairy-Bounes, G. C., and Fischgold, H. (1953). Electroenceph. clin. \& Neurophysiol., 5, 343.

Li, C. L., Jasper, H. and Henderson, L. (1952). Ibid., 4, 513.

Loomis, A. L., Harvey, E. N., and Hobart, G. A. (1937). J. exp. Psychol., 21, 127.

Matthes, K. (1938). J. Neurophysiol., 1, 413. Symposium, ed. Wolstenholme, G. E. W., p. 180 . Churchill, London.

Mosso, A. (1881). Ueber den Kreislauf des Blutes im menschlichen Gehirn. Veit, Leipzig.

Neumann, C. (1943). Amer. J. Physiol., 138, 618. Lhamon, W. T., and Cohn, A. E. (1944). J. clin. Invest., $\overline{\vec{A}}$ 23, 1 .

Pampiglione, G (1952a) Proc Electro-physiol. Technol. Ass 3, 3.

(1952b). Rev. neurol. (Paris), 87, 197 (1953). Vth International Neurological Congress, Lisbon, 1953, Vol. 2, p. 175. Lisbon.

, and Martin, F. (1954). Schweiz. Arch. Neurol., Psych., 71, 277.

Shepard, J. F. (1906). Amer. J. Psychol., 17, 522.

(1914). The Circulation and Sleep. Macmillan, New York

Uhlenbruck, P. (1924). Z. Biol., 80, 35.

Warren, J. V., Walter, C. W., Romano, J., and Stead, E. A. (1942). J. clin. Invest., 21, 665 .'

Wiersma, E. D. (1913). Z. ges. Neurol. Psychiat., Orig, 19, 1. 